\title{
Frequent identical admission-readmission episodes are associated with increased mortality
}

\author{
Authors: Christopher H Fry, ${ }^{\mathrm{A}}$ David Fluck ${ }^{\mathrm{B}}$ and Thang S Han ${ }^{\mathrm{C}}$
}

Frequent emergency readmissions may associate with health consequences. We examined the association between readmissions within 28 days of hospital discharge and mortality in 32,270 alive-discharge episodes (18-107 years). Data collected between 1 April 2017 and 31 March 2019 are presented as age- and sex-adjusted hazard ratios (HR) with 95\% confidence interval (CI).

Compared with no readmission, mortality risk over a 2-year period was increased with one non-identical admission-readmission (AR) episode: $H R=2.4(2.2-2.7)$, two or more non-identical AR episodes: $H R=3.0$ (2.7-3.4), one identical AR episode: $H R=4.7$ (3.6-6.1) and two or more identical AR episodes: $H R=5.0$ (3.8-6.7). Eight conditions associated with AR episodes had increased risk of mortality including congestive heart failure: $\mathrm{HR}=2.7(2.2-3.2)$, chronic pulmonary obstructive disease: $\mathrm{HR}=3.0$ (2.5-3.6), pneumonia: $\mathrm{HR}=2.0$ (1.8-2.3), sepsis: $\mathrm{HR}=2.2$ (1.9-2.5), endocrine disorders: $\mathrm{HR}=1.9$ (1.6-2.3), urinary tract infection: $\mathrm{HR}=1.5$ (1.3-1.7), psychiatric disorders: $\mathrm{HR}=1.5$ (1.1-2.1) and haematological disorders: $\mathrm{HR}=1.5$ (1.2-1.9). Frequent identical AR episodes, particularly from chronic and agerelated conditions, are associated with increased mortality.

KEYWORDS: health economics, healthcare services, readmission prevention, quality of care

DOI: $10.7861 /$ clinmed.2020-0930

\section{Introduction}

Emergency readmission frequency is an indicator of quality of care and cost-efficiency. ${ }^{1-4}$ Early emergency readmissions recorded by the NHS in 2017-2018 showed that there were

Authors: ${ }^{\text {A }}$ chair in applied physiology, School of Physiology, Pharmacology and Neuroscience, University of Bristol, Bristol, UK: ${ }^{B}$ consultant cardiologist, Department of Cardiology, Ashford and St Peter's Hospitals NHS Foundation Trust, Surrey, UK; ' ${ }^{\text {Consultant }}$ endocrinologist, Department of Endocrinology, Ashford and St Peter's Hospitals NHS Foundation Trust, Surrey, UK, and senior lecturer, Institute of Cardiovascular Research, Royal Holloway, University of London, Egham, UK
484,609 emergency readmissions to hospital within 30 days of discharge, a $22 \%$ rise over the previous 5 years. ${ }^{5,6}$ The underlying reasons for this increase in readmission rate remain unclear but the growing population living with age-related chronic conditions ${ }^{7}$ may be a major contributing factor. The cost of emergency readmissions is huge, estimated to be in the order of $\$ 26$ billion per annum in the USA. ${ }^{8}$ Specifically, the cost of readmissions for initial respiratory and cardiac conditions ranges from $\$ 8,500$ to $\$ 9,500$, rising to $\$ 10,000$ for sepsis ${ }^{3}$ and $\$ 13,500$ for coronary artery bypass graft. ${ }^{4}$

Early emergency readmission indicates poor health status of an individual. ${ }^{9-11}$ Hitherto, there has been a paucity of data on frequent readmissions, particularly for the same condition, and its relationship to health consequences. In this study, we aimed to examine the associations of frequent early readmission for the same condition with all-cause mortality, identify conditions presented in the first (index) admission that are most commonly associated with frequent readmissions and relate these frequently readmitted conditions to the risk of mortality.

\section{Methods}

Design, participants and setting

In this 2-year follow-up study, data of consecutive alive-discharge episodes were collected between 1 April 2017 and 31 March 2019 in an NHS hospital. ${ }^{12,13}$

\section{Data procurement}

Index diagnoses presented in the first admission, coded according to the international classification of diseases, ${ }^{14}$ were recorded. Information on the frequency of early readmissions (within 28 days of hospital discharge), mortality within 30 days and 6 months after hospital discharge and over a 2-year period was documented. Cancer and obstetrics admissions were not included, in line with the NHS data collection for emergency hospital admissions. ${ }^{15}$

\section{Definition of types of readmission}

The type of readmission was defined as an 'identical admissionreadmission (AR) episode' or a 'non-identical AR episode', ie readmission either for the same or for a different condition from that of the index admission. For example, if an index admission were for congestive heart failure (CHF) and a readmission were also for $\mathrm{CHF}$ after discharge, then the type of readmission is considered 
as an identical AR episode; a readmission with a condition other than CHF is considered as a non-identical AR episode.

\section{Categorisation of variables}

The frequency of readmissions within 28 days of hospital discharge was categorised into three groups: no readmission, one readmission and two or more readmissions. Five categories were thus created according to the frequency and type of readmissions: no readmission, one non-identical AR episode, two or more non-identical AR episodes, one identical AR episode and two or more identical AR episodes.

\section{Statistical analysis}

Chi-squared tests were used to explore the association between categorical variables. The five classes of frequency and type of readmission were used to predict mortality within 30 days and within 6 months of hospital discharge (an event occurring at a single time point) using multivariable stepwise logistic regression, and to predict mortality over 2 years (a time-dependent event) using multivariable stepwise Cox regression. Data were adjusted for age and sex and presented as odds ratio (OR) and hazard ratio (HR), respectively, with 95\% confidence interval (CI). Analyses were performed using IBM SPSS Statistics, V25.0 (IBM Corp, Armonk, New York).

\section{Results}

\section{Subject characteristics}

A total of 32,270 patients ( 14,878 men and 17,392 women) of mean age $64 \pm 20.5$ years (range 18-107 years) were recruited. Supplementary material S1 shows patient characteristics including primary (index) diagnoses presented in the first admission. The proportions of patients with no readmission, one readmission and two or more readmissions within 28 days of first hospital discharge were $88.5 \%, 8.1 \%$ and $3.3 \%$, respectively. Of those readmitted once, $92.9 \%$ were non-identical AR episodes and $7.1 \%$ were identical AR episodes. Among those readmitted two or more times,
$89.9 \%$ were non-identical AR episodes and $10.1 \%$ were identical AR episodes. There were $2.6 \%, 6.8 \%$ and $10.2 \%$ of patients who died within 30 days, within 6 months and over 2 years post-discharge, respectively. The mean age of death was $81 \pm 12$ years.

\section{Association of frequency and type of readmission with mortality}

Overall, the proportion of patients with no readmission who died was $7.9 \%$. Mortality increased to $25.2 \%, 35.0 \%, 25.5 \%$ and $45.8 \%$ for those with one non-identical AR episode, two or more non-identical AR episodes, one identical AR episode and two or more identical AR episodes, respectively (group differences: $\chi^{2}=1,828, p<0.001$ ).

After adjustment for age and sex, Kaplan-Meier survival plots revealed that the survival probability was lower with increasing frequency of readmission. For a given readmission frequency, an identical AR episode lowered the survival probability further. Thus, individuals at the highest risk of death had the highest frequency of identical AR episodes (Fig 1).

Multivariable Cox regression was conducted to assess mortality within a 2-year period of study. Compared with patients who were not readmitted (reference group), age- and sex-adjusted risk of mortality was increased for those with one non-identical AR episode: HR ( $95 \%$ CI $)=2.4(2.2-2.7)$, two or more non-identical AR episodes: $\mathrm{HR}=3.0$ (2.7-3.4), one identical AR episode: $\mathrm{HR}=$ 4.7 (3.6-6.1) and two or more identical AR episodes: $H R=5.0$ (3.8-6.7) (Table 1). Similar patterns of the association between frequency and type of readmission with mortality within 30 days, and with mortality within 6 months of discharge were also observed (see supplementary material S2).

\section{Association of index admissions with frequency and type of readmission}

From all index admissions examined, higher proportions of frequent readmissions for identical AR episodes were observed
Fig 1. Age- and sex-adjusted KaplanMeier survival curves comparing different categories of frequency and type of readmission.

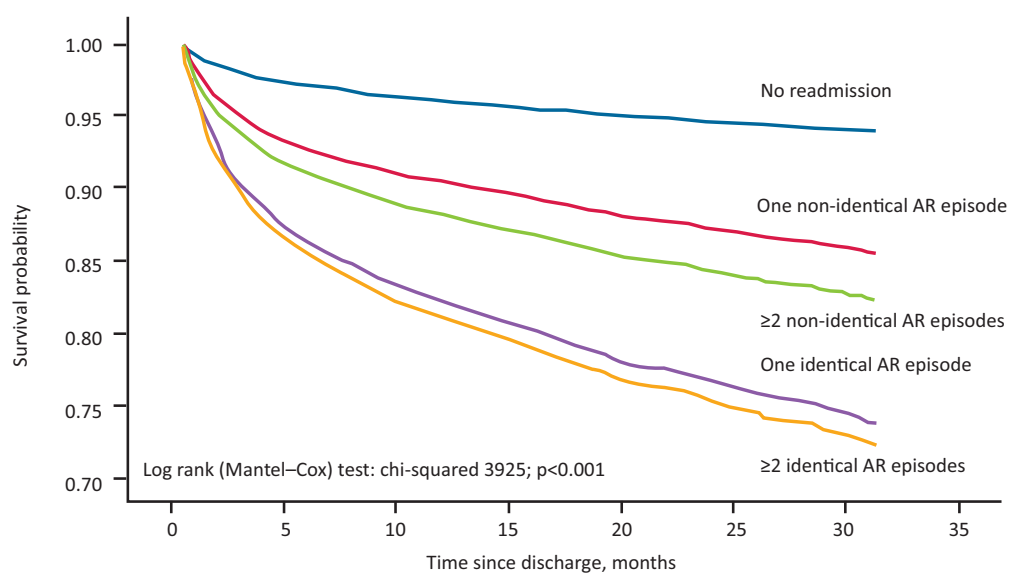

Number at risk

No readmission

One non-identical AR episode

$\geq 2$ non-identical AR episodes

One identical AR episode

$\geq 2$ identical AR episodes
0

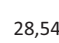

2,476

949

190

107
5

27,303

2,045

723

140

69
$10 \quad 15$

24,592

1,795

614

132

58
15

1,340

445

100

42

$20 \quad 25$

12,762

925

317

76

29

$25 \quad 30$

7,563

533

157

47

21
0

2,386

142

40

18

6
35

0

0

0

0

0 
Table 1. Risk of death over a 2-year period comparing patients who were not readmitted, readmitted once or two times due to a different or the same condition

\begin{tabular}{|c|c|c|c|c|c|c|}
\hline & \multicolumn{6}{|c|}{ Risk of mortality over a 2-year period } \\
\hline & HR & $95 \% \mathrm{CI}$ & $\mathrm{p}$ & HR & $95 \% \mathrm{CI}$ & $\mathrm{p}$ \\
\hline No readmission (reference group) & 1 & - & - & 1 & - & - \\
\hline 1 non-identical AR episode & 3.62 & $3.31-3.95$ & $<0.001$ & 2.43 & $2.22-2.66$ & $<0.001$ \\
\hline$\geq 2$ non-identical AR episodes & 5.32 & $4.74-5.97$ & $<0.001$ & 3.03 & $2.70-3.40$ & $<0.001$ \\
\hline 1 identical AR episode & 4.54 & $3.48-5.91$ & $<0.001$ & 4.69 & $3.60-6.12$ & $<0.001$ \\
\hline$\geq 2$ identical AR episodes & 7.75 & $5.84-10.29$ & $<0.001$ & 5.01 & $3.78-6.66$ & $<0.001$ \\
\hline
\end{tabular}

among five conditions: $\mathrm{CHF}$, chronic obstructive pulmonary disease (COPD), pneumonia (except two or more identical AR episodes), endocrine disorders and urinary tract infection (UTI). Sepsis (7.0\%), psychiatric disorders $(2.2 \%)$ and medical device complications $(4.3 \%)$ peaked at two or more non-identical AR episodes while haematological disorders (3.2\%) and dermatological disorders (7.9\%) peaked at one identical AR episode (Fig 2). There was a trend for a decrease in readmissions for rheumatological disorders, non-specific bodily pain and bone fractures, while the remaining conditions did not associate with frequency and type of readmission (see supplementary material S3).

\section{Association of index admissions with mortality}

Multivariable stepwise Cox regression simultaneously analysed all variables related significantly to higher risk of readmission. This showed that the age- and sex-adjusted mortality over the 2-year period was increased with eight index diagnoses presented on the first admission - CHF: HR = 2.7 (2.2-3.2), COPD: HR = 3.0 (2.53.6), pneumonia: $\mathrm{HR}=2.0$ (1.8-2.3), sepsis: $\mathrm{HR}=2.2(1.9-2.5)$, endocrine disorders: $\mathrm{HR}=1.9$ (1.6-2.3), UTI: HR $=1.5$ (1.3-1.7), psychiatric disorders: $\mathrm{HR}=1.5(1.1-2.1)$ and haematological disorders: HR = 1.5 (1.2-1.9). The adjusted mortality was also increased for any one of the eight index admissions: $\mathrm{HR}=2.0$ (1.8-2.1) (Table 2). The proportion of any one of these eight index admissions for no readmission was $16.7 \%$, rising to $29.5 \%$,

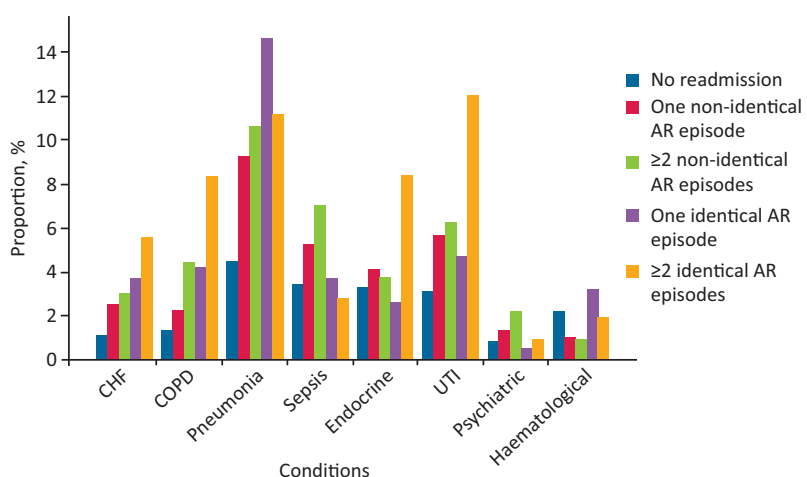

Fig 2. Proportions of eight index admissions in relation to frequency and type of readmission.
$35.2 \%, 33.7 \%$ and $48.6 \%$ for those with one non-identical AR episode, two or more non-identical AR episodes, one identical AR episode and two or more identical AR episodes, respectively (group differences: $\left.\chi^{2}=513, p<0.001\right)$. These eight conditions were also associated with higher risk of death within 30 days and 6 months of hospital discharge (see supplementary material \$4).

Kaplan-Meier survival plots revealed that the survival probability for individuals with any one of the eight conditions related to identical AR episodes (see above) was lower than those who were not admitted with any of these conditions (Fig 3).

\section{Discussion}

In this large study, we found frequent identical admissionreadmission episodes, specifically for chronic or age-related conditions, were associated with increased risk of death within 30 days, within 6 months and over 2 years post-discharge. These findings shed further light on the aetiology of individuals at highest risk of readmissions and mortality, and provide crucial information for healthcare professionals. As far as we are aware, this observation has not been published in the current literature.

We found that the survival probability declined with increasing frequency of readmissions and with identical AR episodes for the same condition. Furthermore, identical AR episodes were an additional risk for lower survival probability for any given frequency of readmission. Thus, individuals who had two or more identical AR episodes for the same condition were at the highest risk of death. These risks could be explained, in part, by the underlying illness of the patient. Using multivariable stepwise logistic regression techniques, eight major conditions were identified as most significantly related to frequent identical AR episodes. Individuals with at least one of these eight conditions were at two to three times greater risk of death within 30 days, within 6 months and over 2 years post-discharge. Of interest, a pattern emerged for identical AR episodes and non-identical AR episode admissions. Frequent identical AR episodes comprised a cluster of chronic conditions and common age-related infections. This raises the possibility that individuals with identical AR episodes represent those with progressive deterioration of a condition towards the end stage. These include CHF, COPD, endocrine disorders (including diabetes), and common infections in older patients such as pneumonia, sepsis and UTI which recur frequently in such individuals due to their decreased ability to recover. By contrast, non-identical AR episodes comprised mostly acute conditions such 
Table 2. Multivariable stepwise Cox regression to assess the risk of mortality over a 2-year period from conditions most commonly associated with frequent emergency readmissions (see supplementary material S3)

\begin{tabular}{|c|c|c|c|c|c|c|}
\hline \multirow[b]{3}{*}{ Died over a 2-year period } & \multicolumn{6}{|c|}{ Risk of mortality } \\
\hline & \multicolumn{3}{|c|}{ Unadjusted } & \multicolumn{3}{|c|}{ Age and sex adjusted } \\
\hline & HR & $95 \%$ CI & $\mathrm{p}$ & HR & $95 \% \mathrm{CI}$ & $\mathrm{p}$ \\
\hline Congestive heart failure & 5.18 & $4.35-6.17$ & $<0.001$ & 2.65 & $2.22-3.15$ & $<0.001$ \\
\hline Chronic obstructive pulmonary disease & 4.21 & $3.53-5.03$ & $<0.001$ & 2.98 & $2.49-3.55$ & $<0.001$ \\
\hline Pneumonia & 3.47 & $3.12-3.87$ & $<0.001$ & 2.02 & $1.82-2.25$ & $<0.001$ \\
\hline Sepsis & 2.47 & $2.14-2.84$ & $<0.001$ & 2.21 & $1.92-2.54$ & $<0.001$ \\
\hline Endocrine disorders & 1.49 & $1.24-1.79$ & $<0.001$ & 1.91 & $1.59-2.30$ & $<0.001$ \\
\hline Urinary tract infection & 2.70 & $2.35-3.11$ & $<0.001$ & 1.45 & $1.25-1.67$ & $<0.001$ \\
\hline Psychiatric disorders & 1.77 & $1.28-2.46$ & 0.001 & 1.54 & $1.11-2.13$ & 0.009 \\
\hline Haematological disorders & 1.37 & $1.08-1.74$ & 0.009 & 1.52 & $1.20-1.93$ & 0.001 \\
\hline Any one of the eight index admissions & 2.69 & $2.51-2.88$ & $<0.001$ & 1.97 & $1.84-2.12$ & $<0.001$ \\
\hline
\end{tabular}

as myocardial infarction, stroke and bone fractures which tended to be managed differently (see below).

Intuitively, it makes sense to accept that individuals with chronic or age-related conditions are inevitably at greater risk of frequent readmissions and mortality. However, there are also individuals with other serious conditions who are not at increased risk for frequent identical AR episodes such as stroke, bone fractures and myocardial infarction. This may be explained by rehabilitation programmes that have been established for these conditions where the level of post-discharge support is more readily available so that patients are less likely to be readmitted within 28 days of discharge. There may be other reasons for some conditions to be associated with recurrent readmission. These include a lack of explanation of the discharge plan provided to the patient; poor execution of discharge instructions and lack of communication with primary care and coordination of care post-discharge, all of which are risk factors for avoidable readmissions. ${ }^{16}$ It is well recognised that the task of reducing hospital readmissions remains challenging in many high-income countries. ${ }^{17,18}$ Further research should focus on safe discharge, ${ }^{19}$ follow-up care for patients $^{20-22}$ and effective communication with primary care physicians. $^{23}$

The identification of eight conditions that carry greater mortality risk with identical AR episodes in this study is consistent with previous studies. These conditions include index diagnosis of $\mathrm{CHF}^{24,25}{ }^{2 O P D},{ }^{24-26}$ pneumonia, ${ }^{25}$ endocrine conditions, ${ }^{26} \mathrm{UTI}^{25}$ and psychiatric disorders. ${ }^{27}$ However, our observation of increased risk of mortality in patients with frequent AR episodes for haematological disorders is novel. Other factors that also associate with readmissions include referral from primary care physicians, deprivation status, male gender, multiple comorbidities, ${ }^{24}$ Ionger initial length of stay in hospital ${ }^{28}$ and older age. ${ }^{24,28}$ Socioeconomic deprivation among patients with sickle cell disease in England is another risk factor for readmissions and inpatient mortality. ${ }^{29}$ We also found older age and male sex to significantly associate with increased risk of readmissions and death.
Fig 3. Age- and sex-adjusted Kaplan-Meier survival curves comparing individuals without against those with any one of the eight index admissions (CHF, COPD, pneumonia, sepsis, endocrine disorders, UTI, psychiatric and haematological disorders).

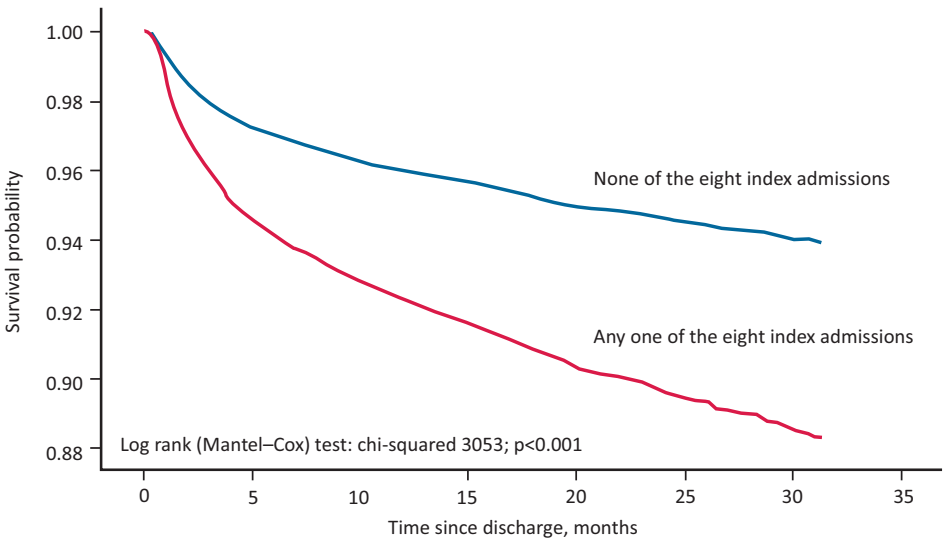

Number at risk

None of the eight index admissions Any one of the eight index admissions

$0 \quad 5$

$25,375 \quad 23,286$

$6,891 \quad 5,760$
10
19,323
4,62 ce discharge, month

$\begin{array}{llllc}15 & 20 & 25 & 30 & 35 \\ 14,077 & 9,192 & 4,512 & 1,076 & 0 \\ 3,294 & 2,075 & 970 & 223 & 0\end{array}$


Further studies would be warranted to examine the level of social and healthcare support for these individuals to reduce readmissions and health consequences. A small pilot study $(n=41)$ of older patients ( $>70$ years) with a post-discharge care bundle, compared to none, showed that fewer patients were readmitted. For those who were, the interval between first admission and any readmission was longer and constituted a lower proportion. ${ }^{30}$ The care bundle consisted of medication reconciliation by a clinical pharmacist, condition-specific education and enhanced discharge planning by a care coordinator, with telephone follow-up. Another study using a similar tool showed over two-thirds of patients, especially those with illiteracy, found communication with a pharmacist to be helpful. ${ }^{31}$ Early readmission rates could even be lowered by a simple telephone contact with patients within 48 hours of discharge compared to those with none. ${ }^{32}$ These studies suggest that specific discharge care bundles are needed to meet the needs of different patient groups, taking into account their age and underlying health conditions. For example, discharge support for young patients with type 1 diabetes would be quite different from those of other groups described above. An important aspect of support for patients with type 1 diabetes is regular surveillance to ensure long-term compliance with insulin treatment. This requires integrated health and social care, involving family members, community healthcare teams including diabetes specialist nurses and general practitioners. ${ }^{33}$ By contrast, older individuals with care needs require hospital-based multidisciplinary teams working with community-based multidisciplinary teams to provide coordinated support through the discharge journey. For those at risk of hospital readmission, the discharge coordinator is responsible for referring to the relevant community-based health and social care practitioners prior to discharge. $^{34}$

The strengths of this study lie in its large number of consecutive adult patients with a wide range of age (18-107 years). This enabled us to estimate the risk of mortality by different categories of frequency and type of readmission that has not been explored in previous studies. Appropriate adjustments were made including age and sex. Characteristics of this study are similar to those of the UK population. ${ }^{12,35,36}$ There are inevitably certain limitations, including the potential loss of patients who might have moved to another area, so that readmissions might have been underestimated, particularly over the 2-year period analysis. Other factors may introduce a bias that will underestimate readmission frequency: for example, some patients who developed a terminal illness would need palliative care instead of hospital readmission. Furthermore, readmission would also be underestimated for those who sustained a hip fracture or stroke and were transferred to rehabilitation.

In conclusion, eight index conditions - namely, CHF, COPD, pneumonia, sepsis, endocrine disorders, UTI, psychiatric and haematological disorders - were identified among individuals with frequent identical AR episodes. Such patients are associated with increased mortality within 30 days and 6 months post-hospital discharge, and over a 2-year period.

\section{Supplementary material}

Additional supplementary material may be found in the online version of this article at www.rcpjournals.org/clinmedicine: S1 - Characteristics of 14,878 men aged 18.0-104.1 years and 17,392 women aged $18.0-106.7$ years.
S2 - Risk of death within 30 days and 6 months of hospital discharge comparing patients who were not readmitted, readmitted once or two times for different or the same condition. S3 - Proportions of patients readmitted with the same condition according to the frequency of admission within 28 days of discharge from hospital.

S4 - Multivariable stepwise logistic regression to assess the risk of death within 30 days and 6 months of hospital discharge from conditions most commonly associated with frequent readmissions (see supplementary material S3).

\section{References}

1 Benbassat ], Taragin M. Hospital readmissions as a measure of quality of health care: advantages and limitations. Arch Intern Med 2000;160:1074-81.

2 Nolte E, Roland M, Guthrie S, Brereton L. Preventing emergency readmissions to hospital: a scoping review. Rand Health Q 2012;2:10.

3 Mayr FB, Talisa VB, Balakumar V, et al. Proportion and cost of unplanned 30-day readmissions after sepsis compared with other medical conditions. JAMA 2017;317:530-1.

4 Shah RM, Zhang Q, Chatterjee $S$ et al. Incidence, cost, and risk factors for readmission after coronary artery bypass grafting. Ann Thorac Surg 2019;107:1782-9.

5 Healthwatch. Emergency readmissions: What's changed one year on? Healthwatch, 2018. www.healthwatch.co.uk/report/201811-14/emergency-readmissions-whats-changed-one-year [Accessed 10 October 2020].

6 Healthwatch. New plans to investigate rising emergency readmissions to hospitals announced in response to concerns raised by patients. Healthwatch, 2019. www.healthwatch.co.uk/news/201901-17/new-plans-investigate-rising-emergency-readmissonshospitals-announced-response [Accessed 10 October 2020].

7 Kingston A, Comas-Herrera A, Jagger C, MODEM project. Forecasting the care needs of the older population in England over the next 20 years: estimates from the Population Ageing and Care Simulation (PACSim) modelling study. Lancet Public Health 2018;3:e447-55.

8 Wilson L. MA patients'readmission rates higher than traditional Medicare, study finds. HealthcareDive, 26 June 2019. www.healthcaredive.com/news/ma-patients-readmission-rates-higher-than-traditional-medicare-study-find/557694/ [Accessed 10 October 2020]

9 Mudireddy P, Scott F, Feathers A, Lichtenstein GR. Inflammatory bowel disease: predictors and causes of early and late hospital readmissions. Inflamm Bowel Dis 2017;23:1832-9.

10 Agrawal S, Garg L, Shah M et al. Thirty-day readmissions after left ventricular assist device implantation in the United States: Insights from the Nationwide Readmissions Database. Circ Heart Fail 2018;11:e004628

11 Lewis KL, Fanaian M, Kotze B, Grenyer BFS. Mental health presentations to acute psychiatric services: 3-year study of prevalence and readmission risk for personality disorders compared with psychotic, affective, substance or other disorders. BJPsych Open 2019;5:e1.

12 Fry $\mathrm{CH}$, Heppleston E, Fluck D, Han TS. Derivation of age-adjusted LACE index thresholds in the prediction of mortality and frequent hospital readmissions in adults. Intern Emerg Med 2020;15:1319-25.

13 Heppleston E, Fry CH, Kelly K et al. LACE index predicts age-specific unplanned readmissions and mortality after hospital discharge. Aging Clin Exp Res 2021;33:1041-8.

14 World Health Organization. CD-10: International statistical classification of diseases and related health problems: Tenth revision. WHO: Geneva, 2004.

15 Digital NHS. NHS Outcomes Framework (NHS OF). https://digital. nhs.uk/data-and-information/publications/statistical/nhs-outcomesframework [Accessed 10 October 2020]. 
16 Aspenson M, Hazary S. The clock is ticking on readmission penalties. Healthc Financ Manage 2012;66:58-63.

17 Wadhera RK, Maddox KEJ, Wasfy JH et al. Association of the Hospital Readmissions Reduction Program with mortality among Medicare beneficiaries hospitalized for heart failure, acute myocardial infarction, and pneumonia. JAMA 2018;320:2542-52.

18 Warchol SJ, Monestime JP, Mayer RW, Chien WW. Strategies to reduce hospital readmission rates in a non-Medicaid-expansion state. Perspect Health Inf Manag 2019;16:1a.

19 Wong SP, Sharda N, Zietlow KE, Heflin MT. Planning for a safe discharge: more than a capacity evaluation. J Am Geriatr Soc 2020;68:859-66.

20 Agostinho JR, Gonçalves I, Rigueira J et al. Protocol-based follow-up program for heart failure patients: impact on prognosis and quality of life. Rev Port Cardiol 2019;38:755-64.

21 Catanach B, Betz ME, Tvrdy C et al. Implementing an emergency department telephone follow-up program for suicidal patients: successes and challenges. Jt Comm J Qual Patient Saf 2019;45:725-32.

22 de Mestral C, Kayssi A, Al-Omran M et al. Home care nursing after elective vascular surgery: an opportunity to reduce emergency department visits and hospital readmission. BMJ Qual Saf 2019;28:901-7.

23 Destino LA, Dixit A, Pantaleoni JL et al. Improving communication with primary care physicians at the time of hospital discharge. It Comm J Qual Patient Saf 2017:43:80-8.

24 Lyratzopoulos G, Havely D, Gemmell I, Cook GA. Factors influencing emergency medical readmission risk in a UK district general hospital: a prospective study. BMC Emerg Med 2005;5:1.

25 Morrissey E, McElnay J, Scott M, McConnell B. Influence of drugs, demographics and medical history on hospital readmission of elderly patients: a predictive model. Clin Drug Investig 2003;23:119-28.

26 Ouslander JG, Diaz S, Hain D, Tappen R. Frequency and diagnoses associated with 7-and 30-day readmission of skilled nursing facility patients to a nonteaching community hospital. J Am Med Dir Assoc 2011;12:195-203.

27 Heslin KC, Weiss AJ. Hospital readmissions involving psychiatric disorders, 2012: Statistical Brief \#189. Agency for Healthcare Research and Quality, 2015.

28 Shalchi Z, Saso S, Li HK, Rowlandson E, Tennant RC. Factors influencing hospital readmission rates after acute medical treatment. Clin Med (Lond) 2009;9:426-30.
29 Aljuburi G, Laverty AA, Green SA et al. Socio-economic deprivation and risk of emergency readmission and inpatient mortality in people with sickle cell disease in England: Observational study. J Public Health (Oxf) 2013;35:510-17.

30 Koehler BE, Richter KM, Youngblood L et al. Reduction of 30-day postdischarge hospital readmission or emergency department (ED) visit rates in high-risk elderly medical patients through delivery of a targeted care bundle. J Hosp Med 2009;4:211-8.

31 Cawthon C, Walia S, Osborn CY, et al. Improving care transitions: the patient perspective. J Health Commun 2012;17:312-24.

32 Vernon D, Brown JE, Griffiths E, Nevill AM, Pinkney M. Reducing readmission rates through a discharge follow-up service. Future Healthc J 2019;6:114-7.

33 Diabetes UK. The National service framework (NSF) for diabetes: Five years on ... are we half way there? www.diabetes.org.uk/ resources-s3/2017-11/five_years_on___are_we_half_way_ there2008.pdf [Accessed 13 February, 2021].

34 National Institute for Health and Care Excellence. Transition between inpatient hospital settings and community or care home settings for adults with social care needs. NICE guideline [NG27]. 2015. www.nice.org.uk/guidance/ng27 [Accessed 13 February 2021].

35 Han TS, Fry CH, Gulli G et al. Prestroke disability predicts adverse poststroke outcome: a registry-based prospective cohort study of acute stroke. Stroke 2020;51:594-600.

36 Royal College of Physicians. Clinical effectiveness and evaluation unit on behalf of the intercollegiate stroke working party. SSNAP January-March 2016. Public Report. www.strokeaudit.org/ Documents/National/AcuteOrg/2016/2016-AOANationalReport. aspx [Accessed 10 October 2020].

Address for correspondence: Dr Thang S Han, Department of Endocrinology, Ashford and St Peter's Hospitals NHS Foundation Trust, Guildford Road, Chertsey, Surrey KT16 OPZ, UK.

Email: thang.han@rhul.ac.uk 